Imperial/TP/95-96/49

gr-qc/9607050

\title{
Symmetries of Decoherence Functionals
}

\author{
S. Schreckenberg凹 \\ Blackett Laboratory \\ Imperial College \\ South Kensington \\ London SW7 2BZ
}

May 1996

Submitted to JMP

\begin{abstract}
The basic ingredients of the 'consistent histories' approach to quantum theory are a space $\mathcal{U P}$ of 'history propositions' and a space $\mathcal{D}$ of 'decoherence functionals'. In this article we consider such history quantum theories in the case where $\mathcal{U P}$ is given by the set of projectors $\mathcal{P}(\mathcal{V})$ on some Hilbert space $\mathcal{V}$. Using an analogue of Wigner's Theorem in the context of history quantum theories proven earlier, we develop the notion of a 'symmetry of a decoherence functional' and prove that all such symmetries form a group which we call 'the symmetry group of a decoherence functional'. We calculate - for the case of history quantum mechanics - some of these symmetries explicitly and relate them to some discussions that have appeared previously.
\end{abstract}

PACS numbers: 03.65.Bz, 03.65.Ca, 03.65.Db

\footnotetext{
${ }^{1}$ email: stschr@ic.ac.uk
} 


\section{Introduction}

The decoherent histories approach to quantum theory has received much attention over the last years. One of its main features is that the notion of a history proposition as an entity is build into the framework for such a theory from the very start. This amounts to an entirely new approach to quantum theory as has been discussed by various authors [1, 2, 3, 4.

In [⿴囗十 Isham argued - by looking at standard quantum mechanics from the point of view of the history programme - that the mathematical structure of such theories is best described by separating the ingredients into a space of history propositions, $\mathcal{U P}$, and a space of decoherence functionals, $\mathcal{D}$, both of which can be specified with the aid of certain algebraic properties. In the case of finite-dimensional quantum mechanics when investigated at a finite sequence of $n$ time-ordered, but otherwise arbitrary, time points $t_{1}<t_{2}<\cdots<t_{n}$, these spaces are given by $\mathcal{U P}=\mathcal{P}\left(\mathcal{V}_{n}\right)$ the set of Schrödinger picture projectors on the $n^{-}$ fold tensor product space $\mathcal{V}_{n}:=\mathcal{H}_{t_{1}} \otimes \mathcal{H}_{t_{2}} \otimes \cdots \otimes \mathcal{H}_{t_{n}}$ of the single time Hilbert spaces $\mathcal{H}_{t_{i}}$. The 'histories', that is time-ordered sequences of Schrödinger picture projection operators $\left(\alpha_{t_{1}}, \alpha_{t_{2}}, \ldots, \alpha_{t_{n}}\right)$ commonly used in the formalism of decoherent histories, are uniquely associated with a subset of $\mathcal{P}\left(V_{n}\right)$, that is given by homogeneous projection operators of the form $\alpha_{h}:=\alpha_{t_{1}} \otimes \alpha_{t_{2}} \otimes \cdots \otimes \alpha_{t_{n}} \in \mathcal{P}\left(\mathcal{V}_{n}\right)$. The classification theorem for decoherence functionals $d \in \mathcal{D}$ [5] shows that-for an arbitrary finite-dimensional Hilbert space $\mathcal{V}$ complex-valued, bounded decoherence functionals $d: \mathcal{P}(\mathcal{V}) \times \mathcal{P}(\mathcal{V}) \rightarrow \mathbb{C}$, which have to fulfill the requirements of

$$
\begin{array}{ll}
\circ & \text { Hermiticity }: d(\alpha, \beta)=d(\beta, \alpha)^{*} \quad \forall \alpha, \beta \in \mathcal{P}(\mathcal{V}) \\
\circ & \text { Positivity }: d(\alpha, \alpha) \geq 0 \quad \forall \alpha \in \mathcal{P}(\mathcal{V}) \\
\circ & \text { Additivity }: d(\alpha \oplus \beta, \gamma)=d(\alpha, \gamma)+d(\beta, \gamma) \\
\circ & \text { Normalisation }: d(1,1)=1
\end{array}
$$

are in one-to-one correspondence with certain operators $X=X_{1}+i X_{2} \in \mathcal{X}_{\mathcal{D}}$ on $\mathcal{V} \otimes \mathcal{V}$ according to the rule:

$$
d(\alpha, \beta)=\operatorname{tr}_{\mathcal{V} \otimes \mathcal{V}}(\alpha \otimes \beta X)
$$

with the restriction that

$$
\begin{array}{ll}
\text { a) } & X^{\dagger}=M X M \quad \text { with } M(|v\rangle \otimes|w\rangle):=|w\rangle \otimes|v\rangle, \quad \forall|v\rangle,|w\rangle \in \mathcal{V} . \\
\text { b) } & \operatorname{tr}_{\mathcal{V} \otimes \mathcal{V}}\left(\alpha \otimes \alpha X_{1}\right) \geq 0 \\
\text { c) } & \operatorname{tr}_{\mathcal{V} \otimes \mathcal{V}}\left(X_{1}\right)=1 .
\end{array}
$$

$\mathcal{X}_{\mathcal{D}}$ denotes the set of all such operators $X_{d}$. In particular this holds true if $\mathcal{V}=\mathcal{V}_{n}$ For standard quantum mechanics - when looked at from the perspective of the history programme - the decoherence functional is associated with an operator

$$
X_{\left(H, \rho_{t_{0}}, \rho_{t_{f}}\right)}=\frac{1}{\operatorname{tr}_{\mathcal{H}}\left(\rho_{t_{0}} \rho_{t_{f}}\left(t_{f}\right)\right)} \tilde{X}_{\left(H, \rho_{t_{0}}, \rho_{t_{f}}\right)}
$$


on $\mathcal{V} \otimes \mathcal{V}$. For homogeneous propositions about histories $\alpha_{h}:=\alpha_{t_{1}} \otimes \alpha_{t_{2}} \otimes \cdots \otimes \alpha_{t_{n}}$ the value of $d_{\left(H, \rho_{t_{0}}, \rho_{t_{f}}\right)}\left(\alpha_{h}, \beta_{h}\right)$ is evaluated to be

$$
\begin{aligned}
d_{\left(H, \rho_{t_{0}}, \rho_{f}\right)}\left(\alpha_{h}, \beta_{h}\right) & \left.=\frac{1}{\operatorname{tr}_{\mathcal{H}}\left(\rho_{t_{0}} \rho_{t_{f}}\left(t_{f}\right)\right)} \operatorname{tr}_{\mathcal{V} \otimes \mathcal{V}}\left(\alpha_{h} \otimes \beta_{h} \tilde{X}_{\left(H, \rho_{t_{0}}, \rho_{f}\right.}\right)\right) \\
& =\frac{1}{\operatorname{tr}_{\mathcal{H}}\left(\rho_{t_{0}} \rho_{t_{f}}\left(t_{f}\right)\right)} \operatorname{tr}_{\mathcal{H}}\left(\tilde{C}_{\alpha_{h}}^{\dagger} \rho_{t_{0}} \tilde{C}_{\beta_{h}} \rho_{t_{f}}\left(t_{f}\right)\right),
\end{aligned}
$$

where the 'class' operator $\tilde{C}_{\alpha_{h}}$ [3] is defined to be

$$
\tilde{C}_{\alpha_{h}}:=\alpha_{t_{1}}\left(t_{1}\right) \alpha_{t_{2}}\left(t_{2}\right) \cdots \alpha_{t_{n}}\left(t_{n}\right)
$$

with $\left\{\alpha_{t_{i}}\left(t_{i}\right):=e^{\frac{i}{\hbar} H\left(t_{i}-t_{0}\right)} \alpha_{t_{i}} e^{-\frac{i}{\hbar} H\left(t_{i}-t_{0}\right)}\right\}$ being the associated Heisenberg picture operators. It is this expression from which the operator $X_{\left(H, \rho_{t_{0}}, \rho_{t_{f}}\right)}$ originally had been derived. The operator $\tilde{X}_{\left(H, \rho_{t_{0}}, \rho_{t_{f}}\right)}$-defined on $\mathcal{V} \otimes \mathcal{V}$-associated with the decoherence functional $d_{\left(H, \rho_{t_{0}}, \rho_{t_{f}}\right)} \in \mathcal{D}$ is given by 6

$$
\begin{aligned}
\tilde{X}_{\left(H, \rho_{t_{0}}, \rho_{t_{f}}\right)} & =\left[U\left(t_{1}, t_{0}\right)^{\dagger} \rho_{t_{0}} U\left(t_{1}, t_{0}\right) \otimes U\left(t_{2}, t_{1}\right)^{\dagger} \otimes \cdots \otimes U\left(t_{n}, t_{n-1}\right)^{\dagger}\right] \\
& \otimes\left[U\left(t_{2}, t_{1}\right) \otimes U\left(t_{3}, t_{2}\right) \otimes \cdots \otimes U\left(t_{n}, t_{n-1}\right) \otimes U\left(t_{f}, t_{n}\right) \rho_{t_{f}} U\left(t_{f}, t_{n}\right)^{\dagger}\right] \\
& \times\left(R_{(n)} \otimes 1_{t_{1}} \otimes 1_{t_{2}} \otimes \cdots \otimes 1_{t_{n}}\right) \\
& \times S_{(2 n)} \\
& \times\left(R_{(n)} \otimes 1_{t_{1}} \otimes 1_{t_{2}} \otimes \cdots \otimes 1_{t_{n}}\right),
\end{aligned}
$$

where the last three lines involve universal operators $R_{(n)}, S_{(2 n)}$ that arise by rewriting products of operators - as they appear in equation (1.5) - in terms of tensor-products via the use of the mathematical identity [5]

$$
\operatorname{tr}_{\mathcal{H}}\left(A_{1} A_{2} \cdots A_{m}\right)=\operatorname{tr}_{\otimes \mathcal{H}^{m}}\left(A_{1} \otimes \cdots \otimes A_{m} S\right)
$$

where $A_{i}$ denote operators on the Hilbert space $\mathcal{H}$ and $S$ represents a certain universal operator on $\otimes \mathcal{H}^{m}$. Therefore, the operators $R_{(n)}, S_{(2 n)}$ are system independent. Thus, this operator $\tilde{X}_{\left(H, \rho_{t_{0}}, \rho_{t_{f}}\right)}$ contains essentialy only the initial and final density operators $\rho_{t_{0}}, \rho_{t_{f}}$ and the time-evolution operator $U\left(t_{i}, t_{i-1}\right)$. For a more detailed account of the formalism used here see [4, 6].

One important result of this separation into a space of history propositions $\mathcal{U P}$ and decoherence functionals $\mathcal{D}$ is that the decoherence functional $d \in \mathcal{D}$ can be thought of as the 'dynamical' content of a history quantum theory. In the case of standard quantum mechanics looked at from the point of view of the history programme one sees explicitly that $d_{-}\left(H, \rho_{t_{0}}, \rho_{t_{f}}\right) i n \mathcal{D}$ carries the knowledge of the initial and final conditions as well as of the Hamiltonian. History propositions $\alpha \in \mathcal{P}(\mathcal{V})$ are given by Schrödinger-picture projection operators and provide thus only the 'kinematical' input of the quantum theory: their 
properties specify the Hilbert space $\mathcal{V}$.

In standard quantum mechanics symmetries of a physical system represented by a Hamiltonian operator $H$ on a Hilbert space $\mathcal{H}$ are often described in terms of operators $A$ on $\mathcal{H}$ that commute with $H$. In order to understand how this comes about it is crucial to dinstinguish in standard quantum mechanics between the notions of a symmetry, a physical symmetry and a symmetry of an operator.

On a complex Hilbert space $\mathcal{H}$ symmetries - as defined by Wigner - are represented by unitary or anti-unitary operators $U$ on $\mathcal{H}$ that are characterized by the property of leaving the modulus of the inner product of any pair of two vectors $|v\rangle,|w\rangle$ invariant, that is

$$
|\langle v, w\rangle|^{2}=|\langle U v, U w\rangle|^{2} .
$$

Wigner's theorem asserts that a physical symmetry - that is an affine one-to-one map on the space of rays in a Hilbert space, that preserves orthogonality between the rays - are in one-to-one correspondence with symmetries and can thus be implemented by a unitary or anti-unitary operator on $\mathcal{H}$.

Given an operator $A$ on $\mathcal{H}$ the symmetries of the operator $A$ are then defined to be all those symmetries $U^{A}$, that commute with $A$, that is $\left[U^{A}, A\right]=0$. As a result, it holds that

$$
|\langle v|A| w\rangle|=\left|\left\langle U^{A} v|A| U^{A} w\right\rangle\right| \quad \forall v, w \in \mathcal{H},
$$

In the case of quantum mechanics, the Hilbert space $\mathcal{H}$ is given by the Hilbert space $\mathcal{H}_{t}$ at a single time-point $t \in \mathbb{R}$. In case that $A$ is given by the unitary evolution operator $U\left(t, t_{0}\right)=e^{-i H\left(t-t_{0}\right)}$ of a quantum mechanical system, the symmetries of this operator are given by all unitary operators $e^{i K}$ on $\mathcal{H}_{t}$ which commute with $U\left(t, t_{0}\right)$. On the level of the (anti-) hermitian operators $K$ and $H$ one requires that $[K, H]=0$.

We would like to understand how such concepts find their place in a theory that places its emphasis on 'history propositions' and 'decoherence functionals'. Recall that the two main ingredients of a history quantum theory are the space of history propositions $\mathcal{U P}$ that - in the case we are considering - is given by the set of projectors $P(\mathcal{V})$ on a finitedimensional Hilbert space $\mathcal{V}$, and the space of decoherence functionals $\mathcal{D}$. Decoherence functionals $d \in \mathcal{D}$ are associated with operators $X_{d} \in \mathcal{X}_{\mathcal{D}}$ on $\mathcal{V} \otimes \mathcal{V}$ and both are intertwined through the expression $d(\alpha, \beta)=\operatorname{tr}_{\mathcal{V} \otimes \mathcal{V}}\left(\alpha \otimes \beta X_{d}\right)$.

In a companion paper [6] we proved an analogue of Wigner's theorem for history quantum theories. We defined the notion of a 'homogeneous symmetry' (HS) and of a 'physical symmetry of a history quantum theory' (PSHQT) and showed that PSHQT are in oneto-one correspondence with HS. Therefore, every PSHQT can be induced by a unitary operator $\hat{U} \otimes \hat{U}$ on $\mathcal{V} \otimes \mathcal{V}$ as follows:

$$
\begin{aligned}
& \mathcal{U P} \times \mathcal{U P} \quad: \quad \alpha \otimes \beta \mapsto \tilde{\alpha} \otimes \tilde{\beta}:=\hat{U} \alpha \hat{U}^{\dagger} \otimes \hat{U} \beta \hat{U}^{\dagger} \quad \forall \alpha \in \mathcal{P}(\mathcal{V}) \\
& \mathcal{X}_{\mathcal{D}} \quad: \quad X_{d} \mapsto X_{\tilde{d}}:=(\hat{U} \otimes \hat{U}) X_{d}\left(\hat{U}^{\dagger} \otimes \hat{U}^{\dagger}\right) .
\end{aligned}
$$


As a consequence of this transformation the invariance

$$
d(\alpha, \beta)=\tilde{d}(\tilde{\alpha}, \tilde{\beta})
$$

for all $d \in \mathcal{D}$ and all $\alpha, \beta \in P(\mathcal{V})$ follows by the property of the trace, see equation (11.2). Thus, a PSHQT possesses the properties of (i) mapping $\mathcal{U P} \times \mathcal{U} \mathcal{P}$ into itself and (ii) mapping $\mathcal{X}_{\mathcal{D}}$ into itself and (iii) leaving the value $d(\alpha, \beta)$ invariant for all $\alpha, \beta \in \mathcal{P}(\mathcal{V})$ and $d \in \mathcal{D}$. Two history quantum theories that are related by a PSHQT are called 'physically equivalent'; this definition turns out to be compatible with the one introduced in [7].

In section 2 we use this analogue of Wigner's theorem to introduce the notion of 'symmetries of a decoherence functional' in analogy to the definition of a symmetry of an operator in standard quantum mechanics. These elements are shown to form a group which we call 'the symmetry group of a decoherence functional'. Furthermore, it is shown-by calculating for history quantum mechanics some of these symmetries explicitly - how this definition seems to capture the mathematical essence of some related discussions that have appeared in the literature [8, 9]. In the closing section 3 we mention some ways one could try to proceed in order to find a satisfactory physical interpretation of the symmetries considered in this article.

\section{Symmetries of Decoherence Functionals}

\subsection{Definition}

Every history quantum theory is determined by the choice of a particular decoherence functional $d \in \mathcal{D}$ that is kept fixed in course of the investigation. We are therefore led to the following definition of symmetries of decoherence functionals:

Definition For a fixed decoherence functional $d \in \mathcal{D}$, the symmetries of $d$ are determined by those unitary or anti-unitary transformations $\hat{U}$ on $\mathcal{V}$, such that

$$
d(\alpha, \beta)=d\left(\hat{U} \alpha \hat{U}^{\dagger}, \hat{U} \beta \hat{U}^{\dagger}\right) \quad \forall \alpha, \beta \in \mathcal{P}(\mathcal{V}) .
$$

The following proposition shows that symmetries of a decoherence functional possess a convenient characterization in terms of commuting operators.

Proposition The set $S_{d}$ of symmetries of a decoherence functional $d \in \mathcal{D}$ is given by

$$
S_{d}:=\left\{\hat{U} \otimes \hat{U} \in \operatorname{Aut}(\mathcal{V} \otimes \mathcal{V}):\left[X_{d}, \hat{U} \otimes \hat{U}\right]=0\right\} .
$$

Thus, for every finite-dimensional Hilbert space $\mathcal{V}, X_{d} \in \mathcal{X}_{\mathcal{D}}$ it holds that :

$$
\left[X_{d}, \hat{U} \otimes \hat{U}\right]=0 \Leftrightarrow \operatorname{tr}_{\mathcal{V} \otimes \mathcal{V}}\left(\alpha \otimes \beta\left[X_{d}-\hat{U}^{\dagger} \otimes \hat{U}^{\dagger} X_{d} \hat{U} \otimes \hat{U}\right]\right)=0 \quad \forall \alpha, \beta \in \mathcal{P}(\mathcal{V}) .
$$




\section{Proof}

Since $X_{d}=X_{1}+i X_{2}$ we have to show commutativity for the real and imaginary part separately. In [6] it was shown that every $X_{1}$ possesses an expansion in terms of operators of the form $P_{\left|e_{i}\right\rangle} \otimes P_{\left|e_{j}\right\rangle}+P_{\left|e_{j}\right\rangle} \otimes P_{\left|e_{i}\right\rangle}$ for some orthonormal basis $\left\{\left|e_{i}\right\rangle\right\}$ of $\mathcal{V}$, and that every $X_{2}$ can be expanded in terms of operators $P_{\left|b_{i}\right\rangle} \otimes P_{\left|b_{j}\right\rangle}-P_{\left|b_{j}\right\rangle} \otimes P_{\left|b_{i}\right\rangle}$ for some-in general-different orthonormal basis $\left\{\left|b_{i}\right\rangle\right\}$ of $\mathcal{V}$.

Thus, for the real part we have to show that

$$
\begin{aligned}
& {\left[P_{\left|e_{i}\right\rangle} \otimes P_{\left|e_{j}\right\rangle}+P_{\left|e_{j}\right\rangle} \otimes P_{\left|e_{i}\right\rangle}, \hat{U} \otimes \hat{U}\right]=0 } \\
\Leftrightarrow & \operatorname{tr}_{\mathcal{V} \otimes \mathcal{V}}\left(\alpha \otimes \beta\left[P_{\left|e_{i}\right\rangle} \otimes P_{\left|e_{j}\right\rangle}+P_{\left|e_{j}\right\rangle} \otimes P_{\left|e_{i}\right\rangle}-\left(P_{\left|e_{i}\right\rangle}^{\hat{U}} \otimes P_{\left|e_{j}\right\rangle}^{\hat{U}}-P_{\left|e_{j}\right\rangle}^{\hat{U}} \otimes P_{\left|e_{i}\right\rangle}^{\hat{U}}\right)\right]\right)=0 \quad \forall \alpha, \beta \in \mathcal{P}(\mathcal{V}),
\end{aligned}
$$

whereas for the imaginary part we must show that

$$
\begin{aligned}
& {\left[P_{\left|b_{i}\right\rangle} \otimes P_{\left|b_{j}\right\rangle}-P_{\left|b_{j}\right\rangle} \otimes P_{\left|b_{i}\right\rangle}, \hat{U} \otimes \hat{U}\right]=0 } \\
& \Leftrightarrow \quad \operatorname{tr}_{\mathcal{V} \otimes \mathcal{V}}\left(\alpha \otimes \beta\left[P_{\left|b_{i}\right\rangle} \otimes P_{\left|b_{j}\right\rangle}-P_{\left|b_{j}\right\rangle} \otimes P_{\left|b_{i}\right\rangle}-\left(P_{\left|b_{i}\right\rangle}^{\hat{U}} \otimes P_{\left|b_{j}\right\rangle}^{\hat{U}}-P_{\left|b_{j}\right\rangle}^{\hat{U}} \otimes P_{\left|b_{i}\right\rangle}^{\hat{U}}\right)\right]\right)=0 \quad \forall \alpha, \beta \in \mathcal{P}(\mathcal{V}) .
\end{aligned}
$$

We first consider the real part of $d \in \mathcal{D}$. By assumption, it is true that

$$
\begin{aligned}
& \operatorname{tr}_{\mathcal{V}}\left(\alpha P_{\left|e_{i}\right\rangle}\right) \operatorname{tr}_{\mathcal{V}}\left(\beta P_{\left|e_{j}\right\rangle}\right)+\operatorname{tr}_{\mathcal{V}}\left(\alpha P_{\left|e_{j}\right\rangle}\right) \operatorname{tr}_{\mathcal{V}}\left(\beta P_{\left|e_{i}\right\rangle}\right) \\
= & \operatorname{tr}_{\mathcal{V}}\left(\alpha P_{\left|e_{i}\right\rangle}^{\hat{U}}\right) \operatorname{tr}_{\mathcal{V}}\left(\beta P_{\left|e_{j}\right\rangle}^{\hat{U}}\right)+\operatorname{tr}_{\mathcal{V}}\left(\alpha P_{\left|e_{j}\right\rangle}^{\hat{U}}\right) \operatorname{tr}_{\mathcal{V}}\left(\beta P_{\left|e_{i}\right\rangle}^{\hat{U}}\right) \quad \forall \alpha, \beta \in \mathcal{P}(\mathcal{V}) .
\end{aligned}
$$

Since this has to hold for all $\alpha, \beta \in \mathcal{P}(\mathcal{V})$ we choose now:

- $\alpha=P_{\left|e_{i}\right\rangle}$ and $\beta=P_{\left|e_{j}\right\rangle}$. Thus,

$$
1=\operatorname{tr}_{\mathcal{V}}\left(P_{\left|e_{i}\right\rangle} P_{\left|e_{i}\right\rangle}^{\hat{U}}\right) \operatorname{tr}_{\mathcal{V}}\left(P_{\left|e_{j}\right\rangle} P_{\left|e_{j}\right\rangle}^{\hat{U}}\right)+\operatorname{tr}_{\mathcal{V}}\left(P_{\left|e_{i}\right\rangle} P_{\left|e_{j}\right\rangle}^{\hat{U}}\right) \operatorname{tr}_{\mathcal{V}}\left(P_{\left|e_{j}\right\rangle} P_{\left|e_{i}\right\rangle}^{\hat{U}}\right)
$$

- $\alpha=P_{\left|e_{i}\right\rangle}=\beta$. Thus

$$
\operatorname{tr}_{\mathcal{V}}\left(P_{\left|e_{i}\right\rangle} P_{\left|e_{i}\right\rangle}^{\hat{U}}\right) \operatorname{tr}_{\mathcal{V}}\left(P_{\left|e_{i}\right\rangle} P_{\left|e_{j}\right\rangle}^{\hat{U}}\right)=0
$$

- $\alpha=P_{\left|e_{j}\right\rangle}=\beta$. Thus

$$
\operatorname{tr}_{\mathcal{V}}\left(P_{\left|e_{j}\right\rangle} P_{\left|e_{j}\right\rangle}^{\hat{U}}\right) \operatorname{tr}_{\mathcal{V}}\left(P_{\left|e_{j}\right\rangle} P_{\left|e_{i}\right\rangle}^{\hat{U}}\right)=0
$$

Assuming that $\operatorname{tr}_{\mathcal{V}}\left(P_{\left|e_{i}\right\rangle} P_{\left|e_{i}\right\rangle}^{\hat{U}}\right)=0$ leads via equation (2.7) to the condition that

$$
1=\operatorname{tr}_{\mathcal{V}}\left(P_{\left|e_{i}\right\rangle} P_{\left|e_{j}\right\rangle}^{\hat{U}}\right) \operatorname{tr}_{\mathcal{V}}\left(P_{\left|e_{j}\right\rangle} P_{\left|e_{i}\right\rangle}^{\hat{U}}\right)
$$

that, since $\hat{U}$ is either unitary or anti-unitary, can only be fulfilled for $\hat{U}$ being a transposition. But, since this has to hold for all orthogonal pairs $\left|e_{i}\right\rangle,\left|e_{j}\right\rangle$, this contradicts the unitarity or anti-unitarity of $\hat{U}$. Therefore, we conclude that $\operatorname{tr}_{\mathcal{V}}\left(P_{\left|e_{i}\right\rangle} P_{\left|e_{j}\right\rangle}^{\hat{U}}\right)=0$. This leads to the condition

$$
1=\operatorname{tr}_{\mathcal{V}}\left(P_{\left|e_{i}\right\rangle} P_{\left|e_{i}\right\rangle}^{\hat{U}}\right) \operatorname{tr}_{\mathcal{V}}\left(P_{\left|e_{j}\right\rangle} P_{\left|e_{j}\right\rangle}^{\hat{U}}\right)
$$


Since $\hat{U}$ is a unitary or anti-unitary operator, it follows that

$$
\left|\operatorname{tr}_{\mathcal{V}}\left(P_{\left|e_{i}\right\rangle} P_{\left|e_{i}\right\rangle}^{\hat{U}}\right)\right| \leq 1 \quad \forall\left|e_{i}\right\rangle \in \mathcal{V}
$$

This shows that the equality (2.11) holds if and only if $\left[P_{\left|e_{i}\right\rangle} \otimes P_{\left|e_{j}\right\rangle}, \hat{U} \otimes \hat{U}\right]=0$. This concludes the proof for the real part.

For the imaginary part we are led to the condition

$$
\begin{aligned}
& \operatorname{tr}_{\mathcal{V}}\left(\alpha P_{\left|b_{i}\right\rangle}\right) \operatorname{tr}_{\mathcal{V}}\left(\beta P_{\left|b_{j}\right\rangle}\right)-\operatorname{tr}_{\mathcal{V}}\left(\alpha P_{\left|b_{j}\right\rangle}\right) \operatorname{tr}_{\mathcal{V}}\left(\beta P_{\left|b_{i}\right\rangle}\right) \\
= & \operatorname{tr}_{\mathcal{V}}\left(\alpha P_{\left|b_{i}\right\rangle}^{\hat{U}}\right) \operatorname{tr}_{\mathcal{V}}\left(\beta P_{\left|b_{j}\right\rangle}^{\hat{U}}\right)-\operatorname{tr}_{\mathcal{V}}\left(\alpha P_{\left|e_{b}\right\rangle}^{\hat{U}}\right) \operatorname{tr}_{\mathcal{V}}\left(\beta P_{\left|b_{i}\right\rangle}^{\hat{U}}\right) \quad \forall \alpha, \beta \in \mathcal{P}(\mathcal{V}) .
\end{aligned}
$$

Since this has to hold for all $\alpha, \beta \in \mathcal{P}(\mathcal{V})$ we choose now:

- $\alpha=P_{\left|b_{i}\right\rangle}$ and $\beta=P_{\left|b_{j}\right\rangle}$. Thus,

$$
1=\operatorname{tr}_{\mathcal{V}}\left(P_{\left|b_{i}\right\rangle} P_{\left|b_{i}\right\rangle}^{\hat{U}}\right) \operatorname{tr}_{\mathcal{V}}\left(P_{\left|b_{j}\right\rangle} P_{\left|b_{j}\right\rangle}^{\hat{U}}\right)-\operatorname{tr}_{\mathcal{V}}\left(P_{\left|b_{i}\right\rangle} P_{\left|b_{j}\right\rangle}^{\hat{U}}\right) \operatorname{tr}_{\mathcal{V}}\left(P_{\left|b_{j}\right\rangle} P_{\left|b_{i}\right\rangle}^{\hat{U}}\right)
$$

Since - by applying Gleason's theorem to the Hilbert space $\mathcal{V} \otimes \mathcal{V}$-it holds that

$$
\left.\operatorname{tr}_{\mathcal{V}}\left(P_{\left|b_{i}\right\rangle} P_{\left|b_{j}\right\rangle}^{\hat{U}}\right) \operatorname{tr}_{\mathcal{V}}\left(P_{\left|b_{j}\right\rangle} P_{\left|b_{i}\right\rangle}^{\hat{U}}\right)=\operatorname{tr}_{\mathcal{V} \otimes \mathcal{V}}\left(P_{\left|b_{i}\right\rangle} \otimes P_{\left|b_{j}\right\rangle} \mid \hat{U}^{\dagger} \otimes \hat{U}^{\dagger}\left(P_{\left|b_{j}\right\rangle} \otimes P_{\left|b_{i}\right\rangle}\right) \hat{U} \otimes \hat{U}\right]\right) \geq 0
$$

it follows that

$$
\operatorname{tr}_{\mathcal{V}}\left(P_{\left|b_{i}\right\rangle} P_{\left|b_{i}\right\rangle}^{\hat{U}}\right) \operatorname{tr}_{\mathcal{V}}\left(P_{\left|b_{j}\right\rangle} P_{\left|b_{j}\right\rangle}^{\hat{U}}\right) \geq 1 \quad \forall i, j \in\{1,2, \ldots, \operatorname{dim} \mathcal{V}\}
$$

But, since $\hat{U}$ is unitary or anti-unitary, the number 1 is the maximum from which we conclude that $\operatorname{tr}_{\mathcal{V}}\left(P_{\left|b_{i}\right\rangle} P_{\left|b_{j}\right\rangle}^{\hat{U}}\right) \operatorname{tr}_{\mathcal{V}}\left(P_{\left|b_{j}\right\rangle} P_{\left|b_{i}\right\rangle}^{\hat{U}}\right)=0$. By the same reasoning as before this leads to $\left[P_{\left|b_{i}\right\rangle} \otimes P_{\left|b_{j}\right\rangle}, \hat{U} \otimes \hat{U}\right]=0$ which concludes the proof for the imaginary part.

\subsection{Discussion}

Since a symmetry of a decoherence functional is represented by a unitary or anti-unitary operator $\hat{U}$, it preserves the algebraic relations among history propositions $\alpha \in \mathcal{P}(\mathcal{V})$. For example, orthogonal elements $\alpha, \beta \in \mathcal{P}(\mathcal{V})$ are mapped into elements $\alpha^{\prime}, \beta^{\prime}$ that are also orthogonal. Therefore, because it also preserves the value of the decoherence functional on pairs of history propositions, consistent sets of $d \in \mathcal{D}$ are mapped into new consistent sets to which the same values of $d \in \mathcal{D}$ are associated. Recall $[10$ that in the formalism used here, consistent sets of history propositions with respect to a particular $d \in \mathcal{D}$ correspond to certain partitions of the unit operator on $\mathcal{V}$ into mutually orthogonal projectors $\left\{\alpha_{i}\right\}_{i=1}^{m \leq \operatorname{dim} \mathcal{V}}$ such that

$$
d\left(\alpha_{i}, \alpha_{j}\right)=\delta_{i j} d\left(\alpha_{i}, \alpha_{i}\right) \quad \forall i, j \in\{1,2, \ldots, m\} .
$$

The properties (1.1) of $d \in \mathcal{D}$ ensure that the values $d\left(\alpha_{i}, \alpha_{i}\right)$ determine a probability distribution on the boolean algebra generated by the $\left\{\alpha_{i}\right\}_{i=1}^{m \leq \operatorname{dim} \mathcal{V}}$. Thus, one can think of 
symmetries of a decoherence functional as a way of generating new consistent sets from given ones. A study of the symmetries of a decoherence functional will therefore reduce the number of algebraic equations representing the consistency requirements.

Since $X_{d}=X_{1}+i X_{2}$, equation (2.2) is equivalent to the two conditions

$$
\left[X_{1}, \hat{U} \otimes \hat{U}\right]=0 \quad \text { and } \quad\left[X_{2}, \hat{U} \otimes \hat{U}\right]=0,
$$

which express the invariance requirement for the real and imaginary part of $X_{d}$ respectively. Since for each $X_{d}$ its real and imaginary part can be expanded with the aid of two, in general, different bases of $\mathcal{V}$, we see that requiring the complex value of $d \in \mathcal{D}$ to be invariant is a much stronger requirement than invariance of the values for the real part $\Re d$ alone. It is interesting to note that the following Corollary holds.

Corollary The following two requirements for the invariance of the real part of the decoherence functionals are equivalent:

$$
\begin{aligned}
\Re d(\alpha, \beta) & =\Re d\left(\hat{U} \alpha \hat{U}^{\dagger}, \hat{U} \beta \hat{U}^{\dagger}\right) \quad \forall \alpha, \beta \in \mathcal{P}(\mathcal{V}) \\
\Leftrightarrow \quad d(\alpha, \alpha) & =d\left(\hat{U} \alpha \hat{U}^{\dagger}, \hat{U} \alpha \hat{U}^{\dagger}\right) \quad \forall \alpha \in \mathcal{P}(\mathcal{V}) .
\end{aligned}
$$

This shows that requiring the invariance of the 'diagonal part' $d(\alpha, \alpha)$ forces the entire real part, $\Re d(\alpha, \beta)$, of $d \in \mathcal{D}$ to be invariant under a symmetry transformation.

\section{Proof}

The proof is similar to the one presented for the proposition. We start with the expression $d(\alpha, \alpha)$. Expand the real part $X_{1}$ of $X_{d}$ in a terms of elementary decoherence functionals $\frac{1}{2}\left(P_{\left|e_{i}\right\rangle} \otimes P_{\left|e_{j}\right\rangle}+P_{\left|e_{j}\right\rangle} \otimes P_{\left|e_{i}\right\rangle}\right)$ with respect to a certain basis $\left\{\left|e_{i}\right\rangle\right\}$ of $\mathcal{V}$. Evaluation of $d(\alpha, \alpha)$ for $\alpha=P_{\left|e_{i}\right\rangle}, \alpha=P_{\left|e_{j}\right\rangle}$ and $\alpha=P_{\left|e_{i}\right\rangle}+P_{\left|e_{j}\right\rangle}$ leads to $\left[X_{1}, \hat{U} \otimes \hat{U}\right]=0$ which equals the condition for the invariance of the values of $\Re d(\alpha, \beta)$ for all $\alpha, \beta \in \mathcal{P}(\mathcal{V})$.

A slightly more subtle observation is the following: Since we are dealing with a unitary operator which preserves the algebraic relations among history propositions, the property of a consistent set of being a partition of unity is always preserved under this mapping. To preserve the value of the decoherence functionals on the elements of the consistent sets, only the 'diagonal' values of $d \in \mathcal{D}, d(\alpha, \alpha)$, have to remain invariant. This leads to the requirement

$$
d(\alpha, \alpha)=d\left(\hat{U} \alpha \hat{U}^{\dagger}, \hat{U} \alpha \hat{U}^{\dagger}\right) \quad \forall \alpha \in \mathcal{P}(\mathcal{V})
$$

which, as we have seen, is not equivalent to the vanishing of the commutator, i.e. $\left[X_{d}, \hat{U} \otimes\right.$ $\hat{U}]=0$. It only leads to the weaker condition

$$
\operatorname{tr}_{\mathcal{V} \otimes \mathcal{V}}\left(\alpha \otimes \alpha X_{d}\right)=\operatorname{tr}_{\mathcal{V} \otimes \mathcal{V}}\left(\alpha \otimes \alpha\left[\hat{U}^{\dagger} \otimes \hat{U}^{\dagger} X_{d} \hat{U} \otimes \hat{U}\right]\right), \quad \forall \alpha \in \mathcal{P}(\mathcal{V})
$$

Therefore, the sets of transformations $\{\hat{U}\}$ obtained by enforcing condition (2.1) or (2.20), can, a priori, be different. It suggests itself to call the transformations determined by 
(2.20) weak symmetries of a decoherence functional, since one only requires the commutator $\left[X_{d}, \hat{U} \otimes \hat{U}\right]$ to vanish weakly, meaning that

$$
\left[X_{d}, \hat{U} \otimes \hat{U}\right]=\Delta_{\hat{U}}
$$

where $\Delta_{\hat{U}}$ is any operator on $\mathcal{V} \otimes \mathcal{V}$, such that

$$
\operatorname{tr}_{\mathcal{V} \otimes \mathcal{V}}\left(\alpha \otimes \alpha \Delta_{\hat{U}}\right)=0, \quad \forall \alpha \in \mathcal{P}(\mathcal{V})
$$

This ensures that condition $(2.20)$ is met. We denote the set of weak symmetries of a decoherence functional by $S_{d}^{w}$. Every $\hat{U} \in S_{d}$ is also a weak symmetry, i.e. $S_{d} \subset S_{d}^{w}$, but the converse is in general not true. In view of the Corollary above, these weak symmetries commute with the real part of $X_{1}$ of $X_{d}$ so that this is a condition on the commutator $\left[X_{2}, \hat{U} \otimes \hat{U}\right]$ between the imaginary part $X_{2}$ and the symmetry transformation.

Weak symmetries possess the property of mapping a consistent set $\left\{\alpha_{i}\right\}$, i.e. a set for which the consistency conditions hold, into a partition of unity $\left\{\alpha_{i}^{\prime}\right\}$, for which $d\left(\alpha_{i}^{\prime}, \alpha_{i}^{\prime}\right)$ defines a probability distribution. But, it has not been shown that this new set is also consistent, i.e. obeys $d\left(\alpha_{i}^{\prime}, \alpha_{j}^{\prime}\right)=0$ for all $i \neq j$. Thus we are led to the question of whether or not the consistency conditions $d\left(\alpha_{i}, \alpha_{j}\right)=0$ determine all partitions of unity $\left\{\alpha_{i}\right\}$ on which $d\left(\alpha_{i}, \alpha_{i}\right)$ defines a probability distribution. Only then can we be sure that weak symmetries map consistent sets into other consistent sets. But this is certainly not the case. One convinces oneself immediately that one should be investigating this question for the consistency condition $\Re d\left(\alpha_{i}, \alpha_{j}\right)=0$, since the vanishing of the imaginary part of the decoherence functional is unimportant in this context. If this question can be answered in the affirmative, this would be a strong argument in favour of using $\Re d\left(\alpha_{i}, \alpha_{j}\right)=0$ as consistency conditions. In that case, the property of definig a probability distribution on a partition of unity would be equivalent to the consistency conditions $\Re d\left(\alpha_{i}, \alpha_{j}\right)=0$.

\subsection{The structure of $S_{d}$}

The purpose of this section is to introduce the notion of a 'symmetry group of $d \in \mathcal{D}$ '.

Definition/Proposition The set $S_{d}:=\left\{\hat{U} \otimes \hat{U} \in \operatorname{Aut}(\mathcal{V} \otimes \mathcal{V}):\left[X_{d}, \hat{U} \otimes \hat{U}\right]=0\right\}$ of symmetries of a decoherence functional $d \in \mathcal{D}$ possesses the structure of a group. This group is called the symmetry group of $d$.

\section{Proof}

The unit element is given by the unit operator $\mathbf{1} \otimes \mathbf{1}$. Multiplication is defined by multiplication of operators. A calculation shows that $\left[X_{d}, \hat{U} \hat{V} \otimes \hat{U} \hat{V}\right]=0$ so that $\hat{U} \hat{V} \otimes \hat{U} \hat{V} \in S_{d}$ whenever $\hat{U} \otimes \hat{U}, \hat{V} \otimes \hat{V} \in S_{d}$. The unique inverse $(\hat{U} \otimes \hat{U})^{-1}$ is given by $\hat{U}^{\dagger} \otimes \hat{U}^{\dagger}$. We have to show that

$$
\hat{U} \otimes \hat{U} \in S_{d} \quad \Longleftrightarrow \quad \hat{U}^{\dagger} \otimes \hat{U}^{\dagger} \in S_{d}
$$


This can be shown as follows.

$$
\begin{aligned}
{\left[X_{d}, \hat{U} \otimes \hat{U}\right]=0 } & \Longleftrightarrow X_{d} \hat{U} \otimes \hat{U}-\hat{U} \otimes \hat{U} X_{d}=0 \\
& \Longleftrightarrow \hat{U}^{\dagger} \otimes \hat{U}^{\dagger} X_{d}^{\dagger}-X_{d}^{\dagger} \hat{U}^{\dagger} \otimes \hat{U}^{\dagger}=0 \\
& \Longleftrightarrow \hat{U}^{\dagger} \otimes \hat{U}^{\dagger} M X_{d} M-M X_{d} M \hat{U}^{\dagger} \otimes \hat{U}^{\dagger}=0,
\end{aligned}
$$

since $X_{d}^{\dagger}=M X_{d} M$. Using the properties $M M=1 \otimes 1$ and $M\left(\hat{U}^{\dagger} \otimes \hat{U}^{\dagger}\right) M=\hat{U}^{\dagger} \otimes \hat{U}^{\dagger}$ one concludes that (2.25) is equivalent to

$$
\left[\hat{U}^{\dagger} \otimes \hat{U}^{\dagger}, X_{d}\right]=0 .
$$

This concludes the proof.

Remark: The inverse is unique since we are looking at unitary operators $\hat{U} \otimes \hat{U}$ on $\mathcal{V} \otimes \mathcal{V}$, even though $\hat{U}$ might be unitary or anti-unitary.

It is interesting to ask for the structure of the set $S_{d}^{w}$ of weak symmetries of d. This set will in general not be closed under the operations of multiplication and taking the inverse as defined above. A quick calculation shows that

$$
\left[X_{d}, \hat{U} \hat{V} \otimes \hat{U} \hat{V}\right]=\Delta_{\hat{U}}(\hat{V} \otimes \hat{V})+(\hat{U} \otimes \hat{U}) \Delta_{\hat{V}}
$$

so that the commutator will, in general, not vanish weakly. It would be interesting to see whether or not for these transformations a kind of 'Dirac-bracket' can be introduced along the following lines:

$$
\left[X_{d}, \hat{U} \otimes \hat{U}\right]^{D}:=\left[X_{d}, \hat{U} \otimes \hat{U}\right]-\Delta_{\hat{U}} .
$$

Thus, it follows that

$$
\left[X_{d}, \hat{U} \hat{V} \otimes \hat{U} \hat{V}\right]^{D}=\Delta_{\hat{U}}(\hat{V} \otimes \hat{V})+(\hat{U} \otimes \hat{U}) \Delta_{\hat{V}}-\Delta_{\hat{U} \hat{V}} .
$$

Defining an operator $\Delta$ on the space of operators by $\Delta(\hat{U} \otimes \hat{U}):=\Delta_{\hat{U}}$, we see that to require

$$
\Delta_{\hat{U}}(\hat{V} \otimes \hat{V})+(\hat{U} \otimes \hat{U}) \Delta_{\hat{V}}-\Delta_{\hat{U} \hat{V}}
$$

to vanish weakly is certainly fulfilled if $\Delta$ possesses the property of being a derivation on the space of transformations $\hat{U} \otimes \hat{U}$. In this case, the set $S_{d}^{w}$ would be closed under multiplication; if $\hat{U}$ is an element of $S_{d}^{w}$, its inverse will, in general, not be an element of $S_{d}^{w}$. It seems that some insight could be gained by such an analysis once a formulation of constraint analysis in the histories formalism is achieved.

\subsection{History Quantum Mechanics}

We want to investigate, for ordinary non-relativistic quantum mechanics when looked at from the perspective of the history programme, whether or not this definition of symmetries is of any value. Again, history propositions $\alpha \in \mathcal{U P}$ are given by projectors 
$\alpha \in \mathcal{P}\left(\mathcal{V}_{n}\right)=\mathcal{P}\left(\otimes_{i=1}^{n} \mathcal{H}_{t_{i}}\right)$. The particular decoherence functional is given by expression (1.7).

Can we find any symmetries $\hat{U}$ on $\mathcal{V}_{n}$ of this $d_{\left(H, \rho_{t_{0}}, \rho_{t_{f}}\right)}$ in the sense specified above?

- Consider a transformation $Q_{t_{i}} \in B\left(\mathcal{H}_{t_{i}}\right), \alpha_{t_{i}} \mapsto Q_{t_{i}} \alpha_{t_{i}} Q_{t_{i}}^{\dagger}$ on all Hilbert spaces $\mathcal{H}_{t_{i}}$ associated with $n$ time-points $\left(t_{1}<t_{2}<\cdots<t_{n}\right)$; choose $\hat{U}:=Q_{t_{1}} \otimes Q_{t_{2}} \otimes \cdots \otimes Q_{t_{n}}$. Then $X_{d}^{\prime} \equiv \hat{U}^{\dagger} \otimes \hat{U}^{\dagger} X_{d} \hat{U} \otimes \hat{U}$ contains the following terms:

$$
\begin{aligned}
& Q_{t_{1}}^{\dagger} U\left(t_{1}, t_{0}\right)^{\dagger} \rho_{t_{0}} U\left(t_{1}, t_{0}\right) Q_{t_{1}} \\
& Q_{t_{i}}^{\dagger} U\left(t_{i}, t_{i-1}\right)^{\dagger} Q_{t_{i-1}} \\
& Q_{t_{i}}^{\dagger} U\left(t_{i}, t_{i-1}\right) Q_{t_{i-1}} \\
& Q_{t_{n}}^{\dagger} U\left(t_{f}, t_{n}\right) \rho_{t_{f}} U\left(t_{f}, t_{n}\right)^{\dagger} Q_{t_{n}}
\end{aligned}
$$

The requirement that $\left[\hat{U} \otimes \hat{U}, X_{\left(H, \rho_{t_{0}}, \rho_{t}\right)}\right]=0$ is certainly fulfilled if we choose the same unitary $Q$ for all times $t_{i}$ and require it to commute with the unitary evolution operator $U\left(t_{i}, t_{i-1}\right)$, that is with the Hamiltonian $H,[Q, H]=0$. Furthermore, we notice that $Q$ has to commute with the initial and final density matrices, i.e. $\left[Q, \rho_{t_{0}}\right]=$ $0=\left[Q, \rho_{t_{f}}\right]$.

These examples of symmetries of the standard decoherence functional are the easiest one can find and are exactly those one obtains by transforming the triple $\left(H, \rho_{t_{0}}, \rho_{t_{f}}\right)$ by an appropriate, fixed unitary transformation on $\mathcal{H}$. One can read them off the form (1.5) of the decoherence functional almost immediately [8, 9]. The virtue of the derivation presented here is that it is associated with a well defined operator $\hat{U}=Q \otimes Q \otimes \cdots \otimes Q, n$ times, on $\mathcal{V}$ which fulfills the requirement of definition (2.2). Thus, a symmetry of a decoherence functional is not just determined by operators $Q$ that commute with the Hamiltonian at a fixed 'time-slice'; they take the initial and final conditions into account and are more to be regarded as 'space-time' symmetries of the system, since we have tied together the properties at different time-slices via the tensor product operation.

The appearance of the commutator of $Q$ with the Hamiltonian $H$ signals that Noether's theorem enters the stage in a somewhat disguised form. An explicit understanding of the precise relationship would certainly further the understanding of symmetries of decoherence functionals as defined here. I give a few remarks in the closing section.

\section{Summary and Outlook}

In this article we used the analogue of Wigner's theorem in the context of history quantum theories [6] in order to define the notion of a 'symmetry of a decoherence functional'. We have seen that these symmetries can be characterized transparently in terms of the vanishing of the commutator $\left[X_{d}, \hat{U} \otimes \hat{U}\right]=0$ between the operator $X_{d}$ uniquely associated 
with $d \in \mathcal{D}$ and the operator $\hat{U} \otimes \hat{U}$ associated with the symmetry transformation. It has been shown that the set of symmetries of a decoherence functional forms a group, called 'the symmetry group of $d \in \mathcal{D}$ '. We calculated explicitly some symmetries for the case of history quantum mechanics which could be related to certain examples discussed in [8, 9].

Physical symmetries of history quantum theories and symmetries of decoherence functionals have now aquired a definite mathematical interpretation as transformations on the space of history propositions and on the space of decoherence functionals. But this does not do justice to the importance that symmetries play in almost every physical theory. One would like to use the results presented here to gain a better insight into the physical meaning of PSHQT. Usually, this means to obtain an interpretation of such symmetries of history quantum theories or decoherence functionals at a classical level; an interpretation which is then happily taken over to the 'quantized' theory. Thus, we are led to try to obtain, for example, history quantum mechanics by a certain process of 'quantization' from a 'classical history theory' for classical mechanics. It turns out that history quantum mechanics can indeed be obtained this way via the aid of a history group [11]. Symmetries of history quantum theories and decoherence functionals then correspond to certain transformations on a 'space of histories'. Details will appear elsewhere.

Even though we gained some insight into the properties of symmetries of decoherence functionals I did not attempt in this paper to tackle questions related to the meaning of 'conserved quantities' in this history formalism. This is a difficult issue since in its conception history quantum theories are timeless. These problems have to some extent been discussed in [8] but a satisfactory explanation remains to be found. While searching

for such an explanation an investigation of properties of symmmetries of a decoherence functional - as proposed in this article - might provide further clues to unravel its full significance.

\section{Acknowledgements}

I would like to thank Professor Dr. C.J. Isham for useful discussions.

\section{References}

[1] R.B. Griffiths. Consistent histories and the interpretation of quantum mechanics. J. Stat. Phys., 36:219-272, 1984.

[2] R. Omnès. Logical reformulation of quantum mechanics. I. Foundations. J. Stat. Phys., 53:893-932, 1988.

[3] M. Gell-Mann and J. Hartle. Quantum mechanics in the light of quantum cosmology. In S. Kobayashi, H. Ezawa, Y. Murayama, and S. Nomura, editors, Proceedings of the Third International Symposium on the Foundations of Quantum Mechanics in the Light of New Technology, pages 321-343. Physical Society of Japan, Tokyo, 1990. 
[4] C.J. Isham. Quantum logic and the histories approach to quantum theory. J. Math. Phys., 35: 2157-2185 (1994).

[5] C.J. Isham, N. Linden and S. Schreckenberg. The classification of decoherence functionals: An analogue of Gleason's theorem. J. Math. Phys., 35: 6360-6370 (1994).

[6] S. Schreckenberg. Symmetry and History Quantum Theory: An Analogue of Wigner's Theorem. Imperial TP/95-96/40

[7] M. Gell-Mann and J. Hartle. Equivalent sets of Histories and Multiple Quasiclassical Domains. gr-qc/9404013.

[8] J. Hartle, R. Laflamme and D. Marolf. Conservation Laws in the Quantum Mechanics of Closed Systems. Phys. Rev. D, 51: 7007-7016 (1995).

[9] F. Dowker and A. Kent. On the Consistent Histories Approach to Quantum Mechanics. gr-qc/9412067

[10] S. Schreckenberg. Completeness of Decoherence Functionals. J. Math. Phys., 36: 4735-4742 (1995).

[11] C.J. Isham and N. Linden. Continuous histories and the history group in generalised quantum theory. J. Math. Phys, 36: 5392-5408 (1995).

[12] V.S. Varadarajan. Geometry of Quantum Theory, VolI; Van Nostrand 1968. 